\title{
Improvement of Insulation Performance of Solid/Gas Composite Insulation with Embedded Electrode
}

\author{
Takahiko Yamashita, Kazuhisa Iwanaga, Tomohiro Furusato, \\ Hiroyuki Koreeda, Tomoyuki Fujishima \\ Nagasaki University \\ Graduate School of Engineering \\ Nagasaki 852-8521, Japan \\ and Junichi Sato \\ Toshiba Corporation Power Systems Company \\ 2-4, Suehiro-cho, Tsurumi-ku \\ Yokohama 230-0045, Japan
}

\begin{abstract}
Solid insulated switchgear (SIS) has been developed as a ubstithtion for medium voltage sulfur hexafluoride $\left(\mathrm{SF}_{6}\right)$ gas insulated switchgears ts moin circuit including a vacuum interrupter is coated with epoxy resin. Therefore, a so/rd/gas insulation system is composed. For further miniaturization and higher stess design of SIS, improvement of the insulation performance is required. In the solid/gas insulation system, the breakdown strength along the surface is lower than, that of solid. Then, the authors investigated the surface insulation performance of the solid/gas insulation system containing an embedded electrode. In the present study, the sizes of the embedded electrode and the solid insulation materia were varied and the partial discharge inception voltage and the surface breakdowh voltage were measured. As a result, it was found that the surface breakdown Noltage varied considerably with the radius of the embedded electrode. From the result, it was deduced that the surface insulation performance could be improved by choosing the size of the embedded electrode appropriately. Moreover, the effects of the thickness of the insulation material and of the surface distance on thesurface insulation performance were clarified.
\end{abstract}

Index Terms - Composite insulation, electric breakdown, stress control, surface discharge, switchgear

\section{INTRODUCTION}

IN an electric power fystem, switchgears are used for changing the circuit conection. They are also charged with a role of isolating aut when an accident occurred in the system. In that cas of switchgears operate so that they hold the fault at a mining area and prevent a spread accident towards the importan equipment. Therefore, higher reliability in opeyation is required to the switchgears.

It has already been 100 years since an open-type switchgear was intyduced. Now, switchgears have become indispensable for the stable supply of electric power. Various types of switchgears have been developed to meet the requirements such as improvement of reliability, size reduction. One of the factors which greatly affected the change of the type of switchgears was the development of the insulation system. Air insulation, oil insulation, sulfur hexafluoride $\left(\mathrm{SF}_{6}\right)$ gas

Manuscript received on 14 May 2015, in final form 29 September 2015, accepted 3 November 2015. insulation and etc. have been developed and employed for the switchgears. In these days, most of the medium voltage switchgears are the cubicle-type low pressure $\mathrm{SF}_{6}$ gas insulated switchgears (C-GIS). However, $\mathrm{SF}_{6}$ gas was designated as one of the targets of emission control in the global warming prevention conference held in 1997 (COP3), then research and development of $\mathrm{SF}_{6}$ gas free systems are advanced actively [16]. In the medium voltage class, the switchgears insulated with high pressure air or nitrogen has been developed [3] On the other hand, research and development of technological components aiming for the realization of a full solid insulated substation is advanced actively in the extra high voltage class. In that substation, bus bars and transformers will be also insulated with solid insulation materials.

Under the situation mentioned above, a solid insulated switchgear (SIS), in which main circuit including a vacuum interrupter are coated with epoxy resin, has been developed [2]. However, improvement of the surface insulation performance 
of the solid/gas composite insulation system is required for the development of higher stress and compact design.

Therefore, the authors investigated the surface insulation performance of the solid/gas composite insulation system. Metal electrode was embedded into the insulation material of epoxy resin. Then, the effect of the embedded electrode on the insulation performance was investigated. In general, coating metal electrode with solid insulation materials is well known as a method of the improvement of the breakdown characteristics [7]. In addition, the stress control effect of embedding an electrode is known. However, little is known about the effect of the embedded electrode on the surface breakdown voltage in air. In the present study, the sizes of the insulation material and the embedded electrode were varied and the partial discharge inception voltage and the surface breakdown voltage were measured. As a result, it was found that the surface breakdown voltage varied considerably with the radius of the embedded electrode. Moreover, the effects of the thickness of the insulation material and of the surface distance were clarified.

\section{EXPECTED EFFECT OF EMBEDDED ELECTRODE}

Figure 1 shows an illustration of a part of the cross section at the moving part of SIS. High voltage electrode is insulated with air and epoxy resin. Therefore, a solid/gas insulation system is formed. High voltage shield is embedded into epoxy resin. The aim of the embedding electrode is to modify the electric field concentration in air around the triple junction of metal, solid and gas. In order to consider the effeet embedded electrode, a simple test model was shown in Figure 2.

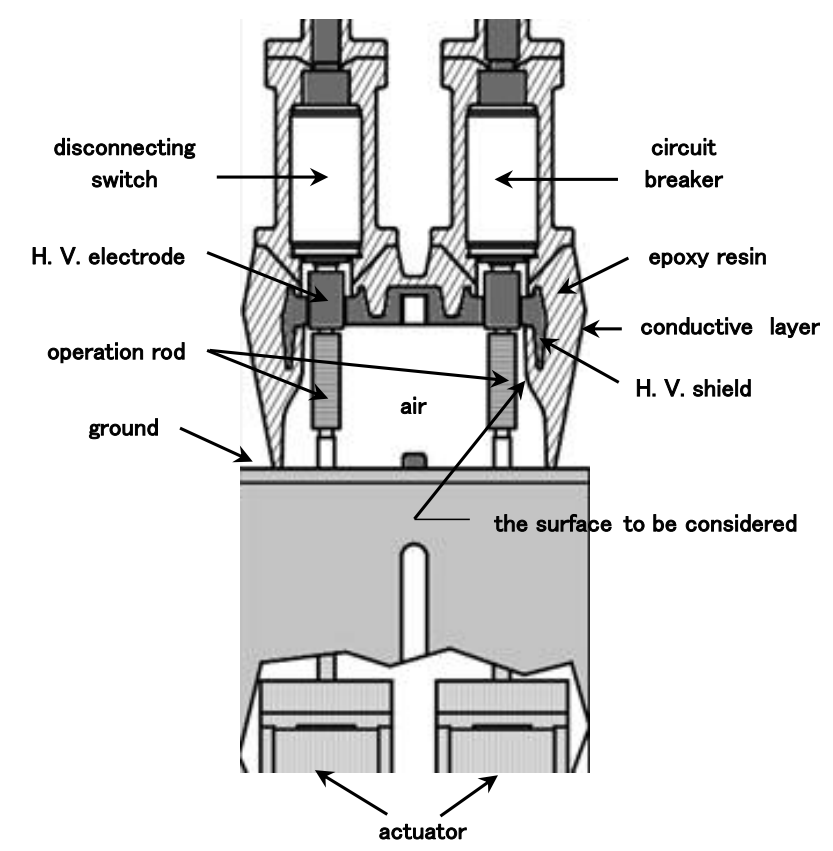

Figure 1. An illustration of a part of the cross section at the moving part of SIS.
Figure 2 shows the cross section of a test sample of the solid/gas composite insulation system. This test sample was the model of insulation system in Figure 1 and was composed of a disc-shaped epoxy resin plate with back-side electrode and disc-shaped embedded electrode. The back-side electrode was formed by painting conductive layer on the underside of the insulation plate. The embedded electrode was made of copper and its thickness was $2 \mathrm{~mm}$. It has round shape at the edge. The radius of curvature was $1 \mathrm{~mm}$. The distance between the bottom of the embedded electrode and the back-side electrode was $4 \mathrm{~mm}$. The embedded electrode was-connected to the center rod electrode which was made of copper with a radius of $5 \mathrm{~mm}$. By embedding electrode, modifieation of the electric stress around the triple junction ioxpected.

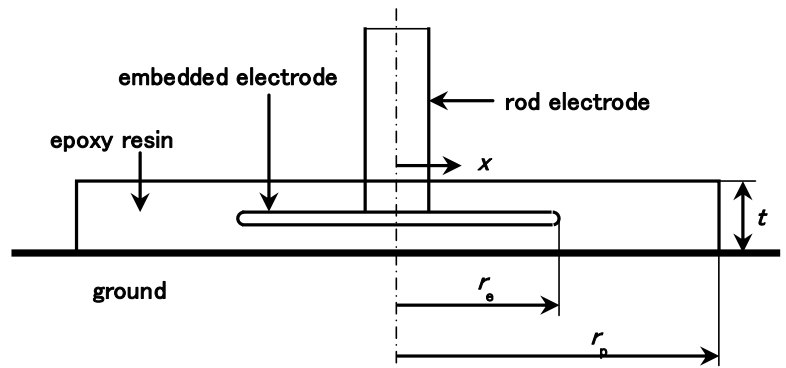

Figure 2. A model of composite insulation system with embedded electrode.

Figure 3 shows an example of the calculation result of the potential distribution. In that case, the radius of the insulation plate, $\boldsymbol{r}_{\mathbf{p}}$ was $100 \mathrm{~mm}$, the thickness of the plate, $\boldsymbol{t}$ was $11 \mathrm{~mm}$, the radius of the embedded electrode, $r$ e was $50 \mathrm{~mm}$, the thickness of the embedded electrode was $2 \mathrm{~mm}$, the thickness between the embedded electrode and the back-side electrode was $4 \mathrm{~mm}$ and the dielectric constant of epoxy resin was 3.4. In Figure 3, modification of the electric field concentration around the triple junction can be seen, as expected before. On the other hand, the electric field concentration around the tip of the embedded electrode can be seen.

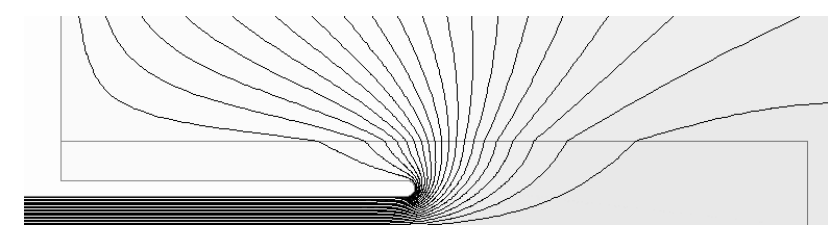

Figure 3. Calculation result of the potential distribution when the static voltage was applied to the rod electrode and the back-side electrode was grounded. $\boldsymbol{r}_{\mathbf{p}}=100(\mathrm{~mm}), \boldsymbol{r}_{\mathrm{e}}=50(\mathrm{~mm})$ and $\boldsymbol{t}=11(\mathrm{~mm})$.

In order to examine the stress control effect of the embedded electrode, $\boldsymbol{r}$ e was varied and the electric field distribution along the surface was calculated by Ansoft Maxwell (Ansoft Japan). The calculation results are shown in Figure 4. The surface position from the center of the rod electrode is expressed with $\boldsymbol{x}$, the voltage of $100 \mathrm{kV}$ was applied to the rod electrode and the back-side electrode was grounded. When $\boldsymbol{r}_{\mathrm{e}}=5(\mathrm{~mm})$, the radius is the same as the 
center rod electrode. Incidentally, the electric field at the triple junction without embedded electrode becomes infinite theoretically.

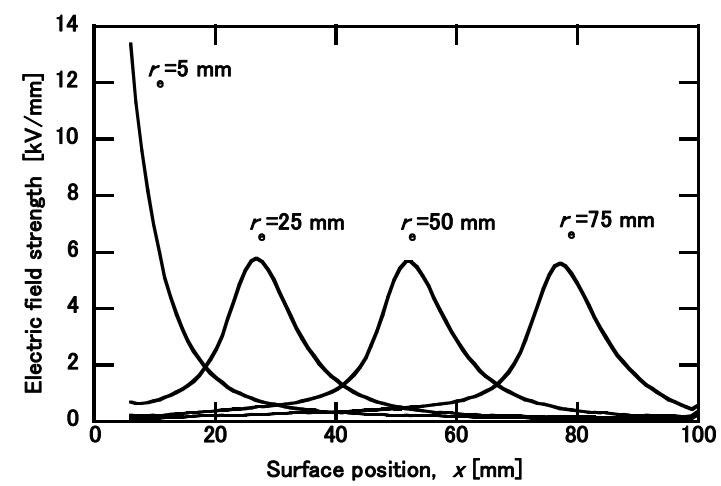

Figure 4. Calculation results of electric field distribution along the surface. $\boldsymbol{r}_{\mathrm{p}}=100(\mathrm{~mm})$ and $\boldsymbol{t}=11(\mathrm{~mm})$.

Compared with the case when $\boldsymbol{r}_{\mathbf{e}}=5(\mathrm{~mm})$, the maximum electric field strength became much smaller when $\boldsymbol{r}_{\mathrm{e}}$ was larger than $25 \mathrm{~mm}$. The position where the electric field strength along the surface was the maximum appeared just above the tip of the embedded electrode. The maximum electric field strength was almost the same regardless of $\boldsymbol{r}_{\mathrm{e}}$ in the range of 25 to $75 \mathrm{~mm}$. The position $\boldsymbol{x}$ with the maximum electric field strength was almost equal to $\boldsymbol{r}$ e.

Discharge inception on the surface is considered to be related to the electric field strength along the surface, increase of the discharge inception voltage is expected by embedding the electrode more than $25 \mathrm{~mm}$ in radius. The uniformity of the electric field is also improved. Then, the breakdown voltage is expected to increase. On theother hand, by moving the position of the maximum electric field strength towards the edge of the insulation plate, the distance between the position of the maximum electric field strength and the ground electrode along the surface decreases. Then, the breakdown voltage is considered to vary with the radius of the embedded electrode. The reason th the surface potential distribution is varied with $\boldsymbol{r}_{\text {and }}$ the propagation of the surface discharge, that is the elongation of the partial discharge, will depend on the surface potential distribution.

\section{EXPERMMENTAL SET-UP AND PROCEDURE}

\subsection{TEST SAMPLE}

As shown in Figure 2, the test sample was composed of a disc-shaped epoxy resin plate with back-side electrode and embedded electrode. The radius of the embedded electrode $\boldsymbol{r}_{\mathbf{e}}$, the radius of the disc-shaped insulation plate $\boldsymbol{r} \mathbf{p}$ or the thickness of the plate $\boldsymbol{t}$ was varied according to the purpose of tests, as follows.

\section{(1) Effect of the size of the embedded electrode}

In order to examine the effect of the radius of the embedded electrode, $\boldsymbol{r}_{\mathbf{p}}$ and $\boldsymbol{t}$ were fixed at 100 and $11 \mathrm{~mm}$, respectively, and $\boldsymbol{r}_{\mathrm{e}}$ was varied from 5 to $70 \mathrm{~mm}$. The specifications of the test samples in this case were listed in Table 1.

Table 1. Specifications of test samples used for the test of the effect of the radius of the embedded electrode.

\begin{tabular}{c|c|c}
\hline $\begin{array}{c}\text { Radius of the } \\
\text { Plate }(\mathrm{mm})\end{array}$ & $\begin{array}{c}\text { Thickness of the } \\
\text { Plate }(\mathrm{mm})\end{array}$ & $\begin{array}{c}\text { Radius of the } \\
\text { Embedded Electrode } \\
(\mathrm{mm})\end{array}$ \\
\hline 100 & 11 & 5 \\
100 & 11 & 15 \\
100 & 11 & 25 \\
100 & 11 & 50 \\
100 & 11 & 70 \\
\hline
\end{tabular}

(2) Effect of the thickness of the insulation plate

In order to examine the effect of the hickness of the insulation material, $\boldsymbol{r}_{\mathbf{p}}$ and $\boldsymbol{r}_{\mathrm{e}}$ were fixed at 100 and $50 \mathrm{~mm}$, respectively, and $\boldsymbol{t}$ was varieofrom $/ 9$ to $21 \mathrm{~mm}$. The specifications of the test samples in this case were listed in Table 2.

Table 2. Specifications of test samples used for the test of the effect of the thickness of the insulation plate.

\begin{tabular}{c|c|c}
\hline $\begin{array}{c}\text { Radius of the } \\
\text { Plate }(\mathrm{mm})\end{array}$ & $\begin{array}{c}\text { Thickness of the } \\
\text { Plate }(\mathrm{mm})\end{array}$ & $\begin{array}{c}\text { Radius of the } \\
\text { Embedded Electrode } \\
(\mathrm{mm})\end{array}$ \\
\hline 100 & 9 & 50 \\
100 & 11 & 50 \\
100 & 15 & 50 \\
100 & 21 & 50 \\
\hline
\end{tabular}

(3) Effect of the radius of the surface distance

In order to examine the effect of the radius of the surface distance, $\boldsymbol{t}$ and $\boldsymbol{r}_{\mathrm{e}}$ were fixed at 11 and $25 \mathrm{~mm}$, respectively, and $\boldsymbol{r}_{\mathbf{p}}$ was varied from 50 to $250 \mathrm{~mm}$. The specifications of the test samples in this case were listed in Table 3.

Table 3. Specifications of test samples used for the test of the effect of the radius of the insulation plate.

\begin{tabular}{c|c|c}
\hline $\begin{array}{c}\text { Radius of the } \\
\text { Plate }(\mathrm{mm})\end{array}$ & $\begin{array}{c}\text { Thickness of the } \\
\text { Plate }(\mathrm{mm})\end{array}$ & $\begin{array}{c}\text { Radius of the } \\
\text { Embedded Electrode } \\
(\mathrm{mm})\end{array}$ \\
\hline 50 & 11 & 25 \\
100 & 11 & 25 \\
250 & 11 & 25 \\
\hline
\end{tabular}

\subsection{EXPERIMENTAL CIRCUIT AND PROCEDURE}

By changing the radius of the embedded electrode, ac and impulse breakdown tests were conducted. In the present study, the insulation performance was discussed so the minimum breakdown voltage was measured.

\section{(1) AC Tests}

Figure 5 shows the test circuit of ac tests. Using an ac high voltage source, ac high voltage was applied to the center rod electrode and the back-side electrode was grounded. The partial discharge inception voltage (PDIV) and the minimum breakdown voltage (BDV) were measured by a voltage rising method. The voltage rising rate was approximately $1 \mathrm{kV}_{\mathrm{rms}} / \mathrm{s}$. Breakdown was detected from the disruption of the voltage 
waveform observed with a voltage divider and a digital oscilloscope. The partial discharge was detected with a partial discharge detector (Nihonkeisokuki, CD-5). A digital camera was installed above the insulation plate and the state of the partial discharge was observed. The tests were carried out 3 to 4 times for each test sample.

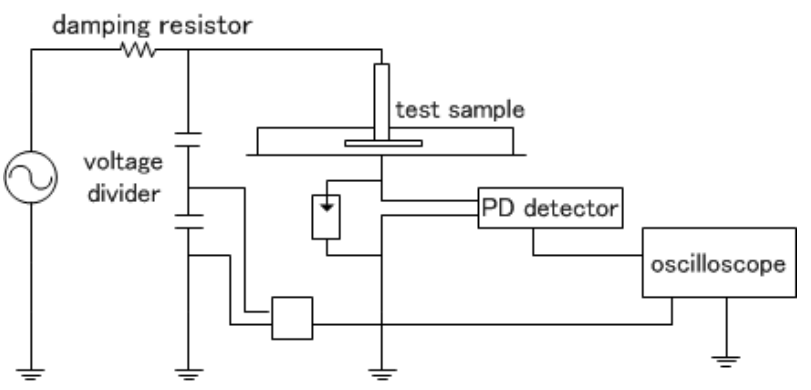

Figure 5. AC test circuit.

(2) Impulse Tests

Figure 6 shows the test circuit of impulse tests. Impulse voltage of $1.2 / 50 \mu \mathrm{s}$ generated by an impulse generator was applied to the center rod electrode and the back-side electrode was grounded through a current detecting resistance. The tests were done at least 20 times for each polarity and test sample. After the first test, the voltage raising method was started from the voltage $80 \%$ of the previous minimum breakdown voltage and stopped at that value.

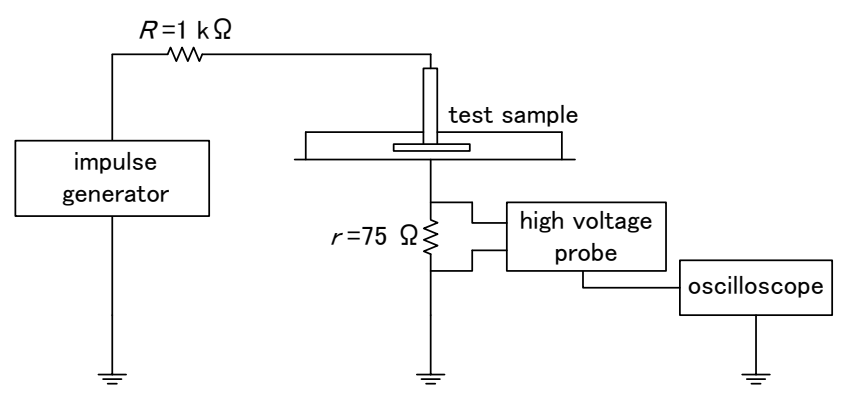

Figure 6. Impulse test circuit.

\section{EXPERIMENTAL RESULTS}

Prior to the measurements of the PDIV and BDV, prebreakdown phenmena were investigated. In addition, the partjalischarge inception position was measured.

\section{THE POSITION OF DISCHARGE INCEPTION}

Figure 7 shows the photographs of the test sample and the partial discharge. An example of the partial discharge in Figure $7 \mathrm{~b}$ was observed under ac voltage and $\boldsymbol{r}_{\mathrm{e}}=50(\mathrm{~mm})$. The exposure time of the digital camera was 10 seconds. Therefore, photograph of the temporal and spatial integration of the partial discharge was taken. Ring-shaped partial discharge was observed as shown in Figure $7 \mathrm{~b}$. From the figure, the position of the partial discharge inception was

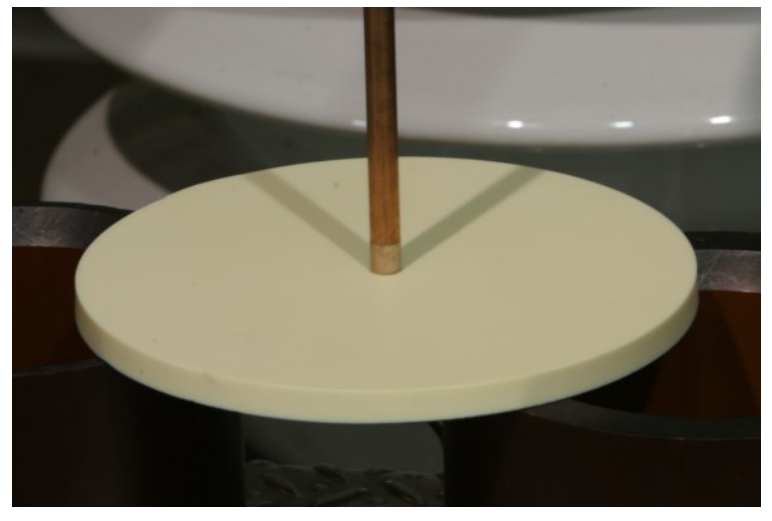

(a) Test sample

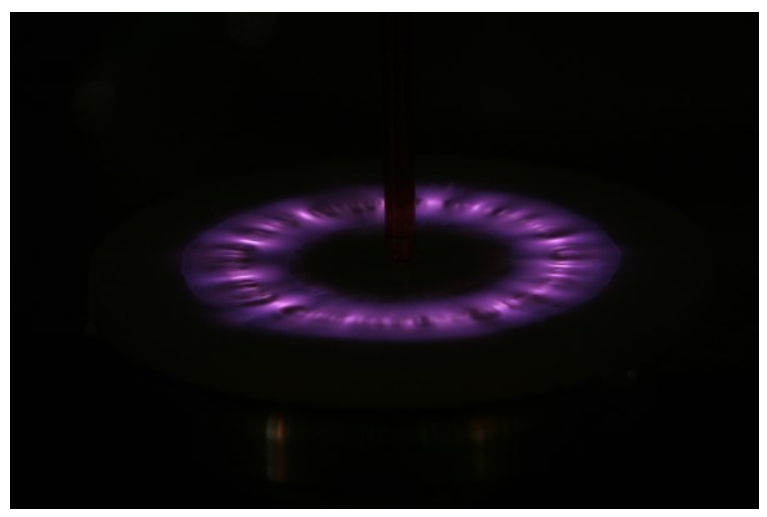

(b) Partial discharge

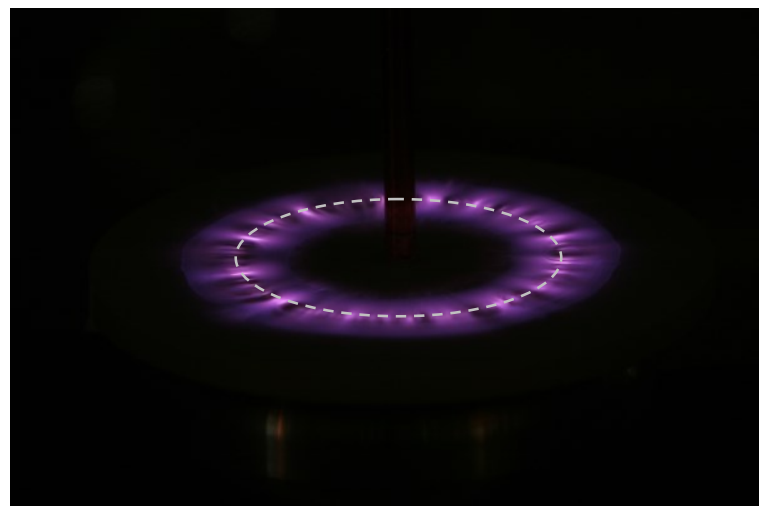

(c) Position of partial discharge inception

Figure 7. Partial discharge under ac voltage. $\boldsymbol{r}_{\mathrm{p}}=100(\mathrm{~mm}), \boldsymbol{r}_{\mathrm{e}}=50(\mathrm{~mm})$, $\boldsymbol{t}=11(\mathrm{~mm})$ and the applied voltage was $47 \mathrm{k} V_{\text {rms. }}$.

estimated as the point where the light emission intensity was the highest, as shown in Figure 7c by a circle of broken line. PDIV was the lowest voltage at which the partial discharge could be observed. The voltage and the radius of the circle at the partial discharge inception were measured.

Figure 8 shows the relation between the radius of the circle mentioned above and $\boldsymbol{r}$ e. The partial discharge inception position was almost the same as $\boldsymbol{r}$ e. The electric field strength along the surface became the maximum at the point just above the tip of the embedded electrode, as shown in Figure 4. Therefore, it is deduced that the partial discharge is initiated at 


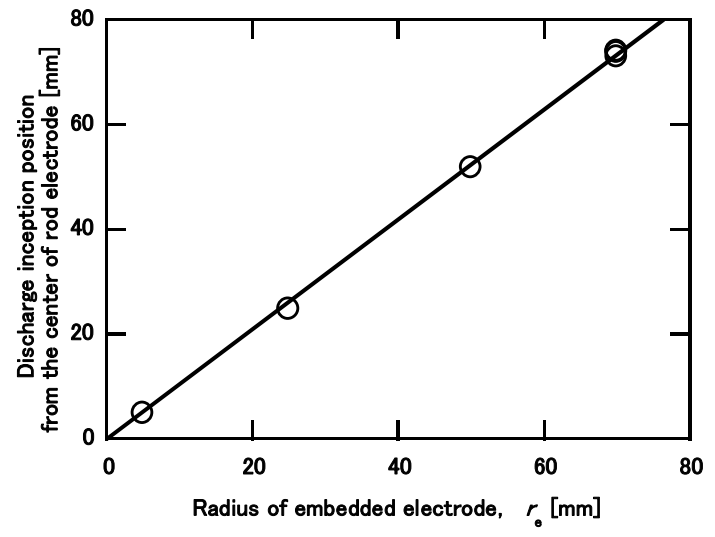

Figure 8. Relation between the partial discharge inception position and the radius of the embedded electrode. $\boldsymbol{r}_{\mathbf{p}}=100(\mathrm{~mm})$ and $\boldsymbol{t}=11(\mathrm{~mm})$.

the point of the maximum electric field strength along the surface.

In case of impulse voltage application, the light emission from the partial discharge was quite weak. Then, an image intensifier (Hamamatsu Photonics, Night Viewer C3100) was employed to observe the partial discharge. As a result, the partial discharge could just be seen around the position of the maximum electric field strength. However, photographs could not be taken.

\subsection{AC BREAKDOWN}

Figure 9 shows the relation between ac breakdown voltag (BDV) and the radius of embedded electrode, $\boldsymbol{r}_{\mathrm{e}}$. In this fig we, partial discharge inception voltage (PDIV) was aso showi. BDV and PDIV are expressed in peak value. BDV axied yith $\boldsymbol{r}$ e. It increased with $\boldsymbol{r}_{\mathrm{e}}$ in the range of 5 to $50 \mathrm{~mm}$. $\boldsymbol{o n}_{\mathrm{n}}$ the other hand, it decreased in the range larger than $00 \mathrm{~mm}$.

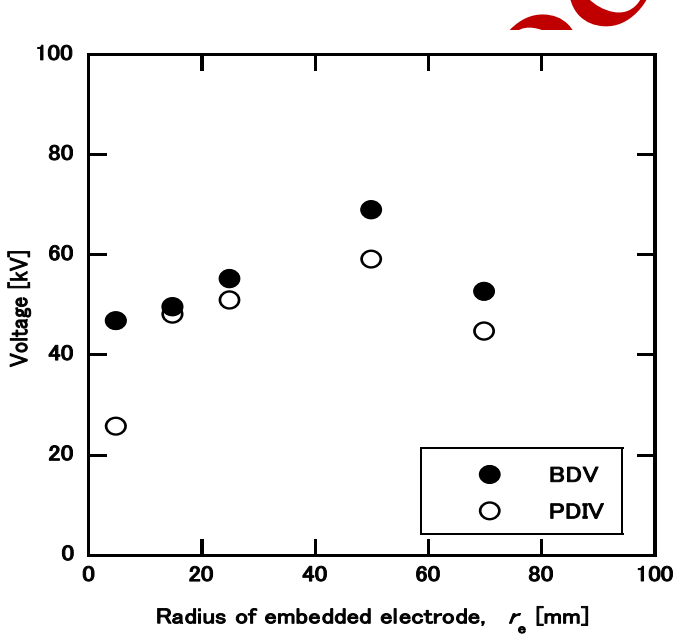

Figure 9. Relation between ac breakdown voltage and the radius of the embedded electrode. $\boldsymbol{r}_{\mathbf{p}}=100(\mathrm{~mm})$ and $\boldsymbol{t}=11(\mathrm{~mm})$.

BDV and PDIV had maximum values when $\boldsymbol{r}_{\mathrm{e}}=50(\mathrm{~mm})$. The maximum BDV was almost 1.5 times higher compared with the case $\boldsymbol{r}_{\mathrm{e}}=5(\mathrm{~mm})$ which was the same as the radius of the center rod electrode. Therefore, it is deduced that ac breakdown voltage can be increased if the radius of the embedded electrode is chosen appropriately.

In case of ac, PDIV is also important for the solid/gas composite insulation system. If the partial discharge occurs under the service voltage it may lead to the deterioration of the solid insulation material. AC partial discharge inception voltage also can be increased if the radius of the embedded electrode is chosen appropriately.

Figure 10 shows ac BDV and PDIV measured by granging the thickness of the disc-shaped insulation plate, $t$. BDV and PDIV increased with $\boldsymbol{t}$. From this figure, it is deduced that increasing the thickness of the solid insulation paterial is effective for the improvement of the $\mathrm{Asurface}$ insulation performance.

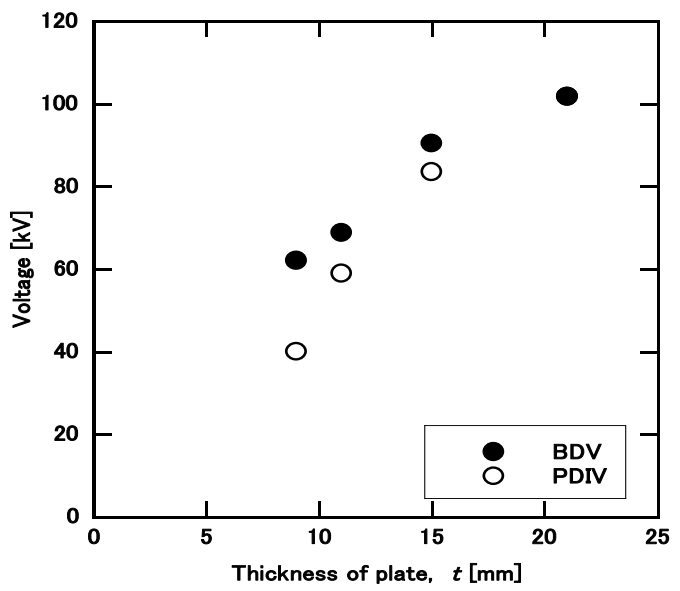

Figure 10. Relation between ac breakdown voltage and the thickness of the insulation plate. $\boldsymbol{r}_{\mathrm{p}}=100(\mathrm{~mm})$ and $\boldsymbol{r}_{\mathrm{e}}=50(\mathrm{~mm})$.

Figure 11 shows ac BDV and PDIV measured by changing the radius of the disc-shaped insulation plate, $\boldsymbol{r}$ p. BDV increased with $\boldsymbol{r}$ p. However PDIV was almost the same regardless of $\boldsymbol{r}_{\mathbf{p}}$. Therefore, the effect of increasing the surface distance on the surface insulation performance is considered to be limited.

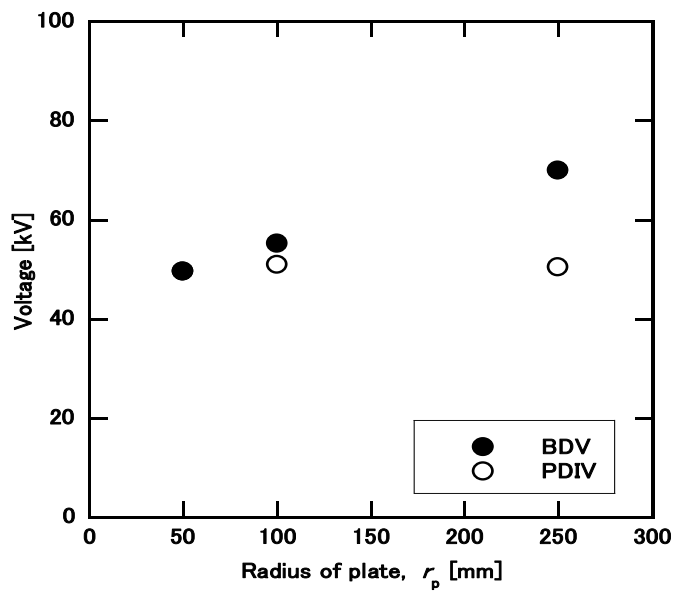

Figure 11. Relation between ac breakdown voltage and the radius of the plate. $\boldsymbol{r}_{\mathrm{e}}=25(\mathrm{~mm})$ and $\boldsymbol{t}=11(\mathrm{~mm})$. 


\subsection{IMPULSE BREAKDOWN}

Impulse voltage of positive or negative polarity was applied to the center rod electrode and the back-side electrode was grounded. In case of impulse voltage, as light emission from the partial discharge was quite weak, photomultiplier (Hamamatsu Photonics, 1P21) was also used to detect the partial discharge. Prior to every voltage application the surface of the test sample was swept with a grounded brush-shaped copper wire bundle in order to remove the charge accumulated on the surface.

Figures 12 and 13 show the test results obtained by changing the radius of the embedded electrode, $\boldsymbol{r}_{\mathrm{e}}$. PDIV was also shown in the figures. Although the variation of impulse BDV was similar to that of ac, impulse BDV was slightly higher than that of ac. And the radius of embedded electrode with the maximum BDV was different between impulse and ac. The maximum breakdown voltage was measured when the radius was $25 \mathrm{~mm}$. In addition, the polarity effect can be seen slightly. Impulse BDV of positive polarity was slightly lower than that of negative polarity.

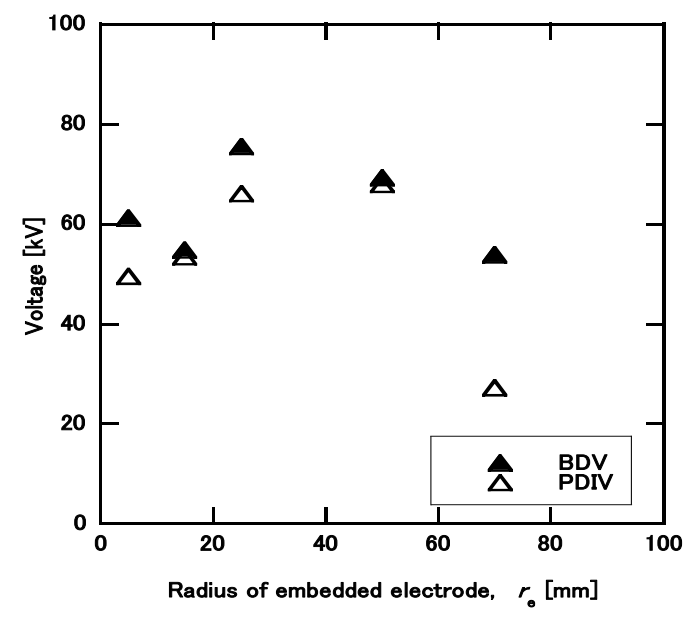

Figure 12. Relation between positive impulse breakdown voltage and the radius of the embedded electrode. $\boldsymbol{r}_{\mathbf{p}}=100(\mathrm{~mm})$ and $\boldsymbol{t}=11(\mathrm{~mm})$.

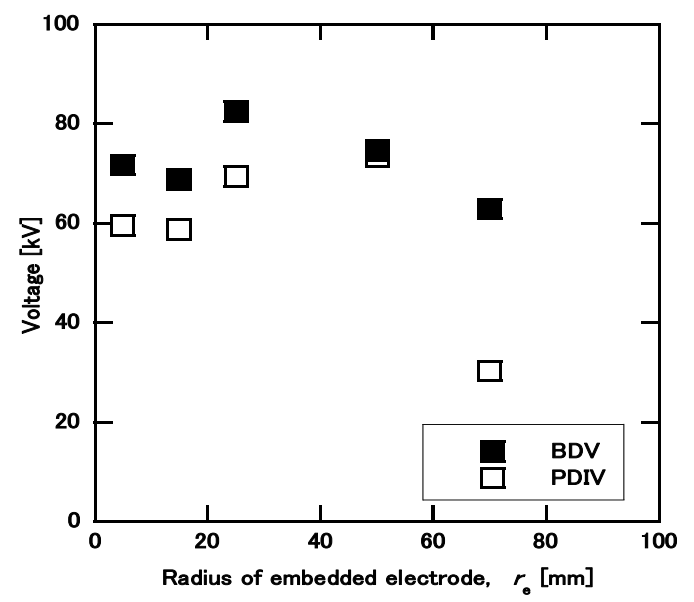

Figure 13. Relation between negative impulse breakdown voltage and the radius of the embedded electrode. $\boldsymbol{r}_{\mathrm{p}}=100(\mathrm{~mm})$ and $\boldsymbol{t}=11(\mathrm{~mm})$.
The variation of impulse PDIV is similar to ac PDIV. However, impulse PDIV is slightly larger than that of ac except for the case of large $\boldsymbol{r}_{\mathrm{e}}$.

Figures 14 and 15 show the test results obtained by changing the thickness of the disc-shaped insulation plate, $\boldsymbol{t}$. BDV increased with $\boldsymbol{t}$. From this figure, it is deduced that increasing the thickness of solid insulation material is effective for improvement of the surface insulation performance.

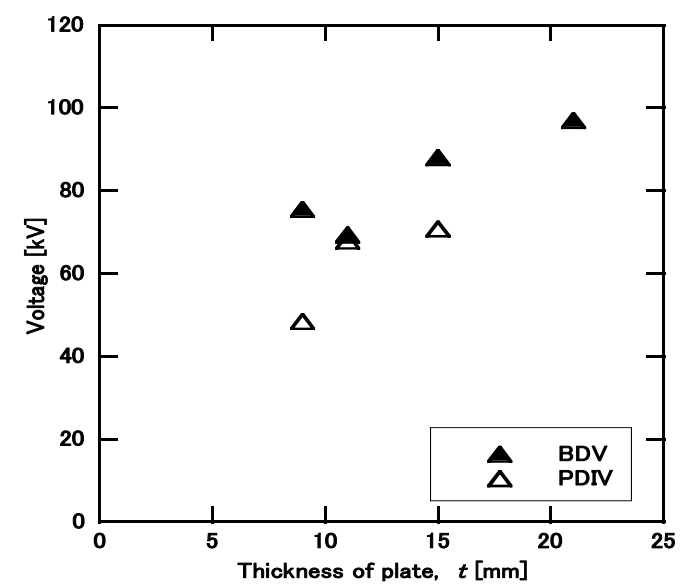

Figure 14. Relation between positive impulse breakdown voltage and the thickness of the insulation plate. $r_{\mathrm{p}}=100(\mathrm{~mm})$ and $\boldsymbol{r}_{\mathrm{e}}=50(\mathrm{~mm})$.

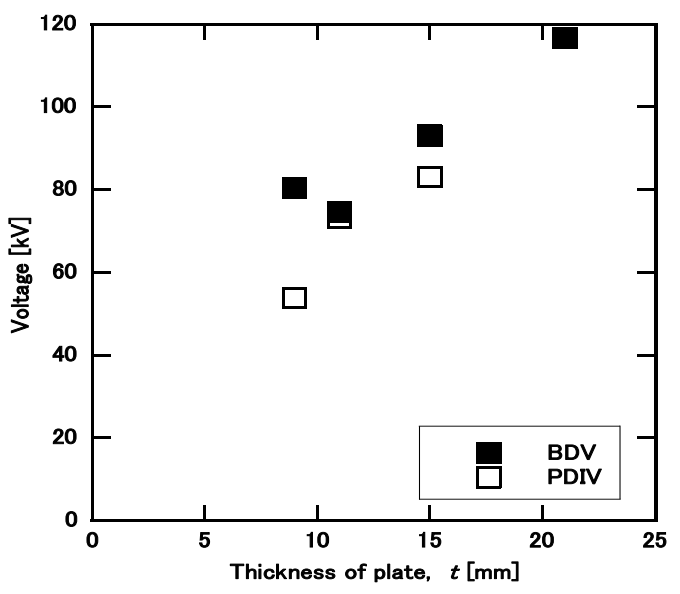

Figure 15. Relation between negative impulse breakdown voltage and the thickness of the insulation plate. $\boldsymbol{r}_{\mathrm{p}}=100(\mathrm{~mm})$ and $\boldsymbol{r}_{\mathrm{e}}=50(\mathrm{~mm})$.

Figures 16 and 17 show the test results obtained by changing the radius of the disc-shaped insulation plate, $\boldsymbol{r}_{\mathbf{p}}$. Compared with the case when $\boldsymbol{r}_{\mathbf{p}}=50(\mathrm{~mm})$, BDV when $\boldsymbol{r}_{\mathbf{p}}=$ $100(\mathrm{~mm})$ was larger, however, BDV when $\boldsymbol{r}_{\mathbf{p}}=250(\mathrm{~mm})$ was almost comparable. Moreover, PDIV when $\boldsymbol{r}_{\mathbf{p}}=250(\mathrm{~mm})$ was much smaller compared with the other cases. Therefore, the effect of increasing the surface distance on the surface insulation performance is considered to be limited.

In all cases, impulse BDV of positive polarity was lower than that of negative polarity. 


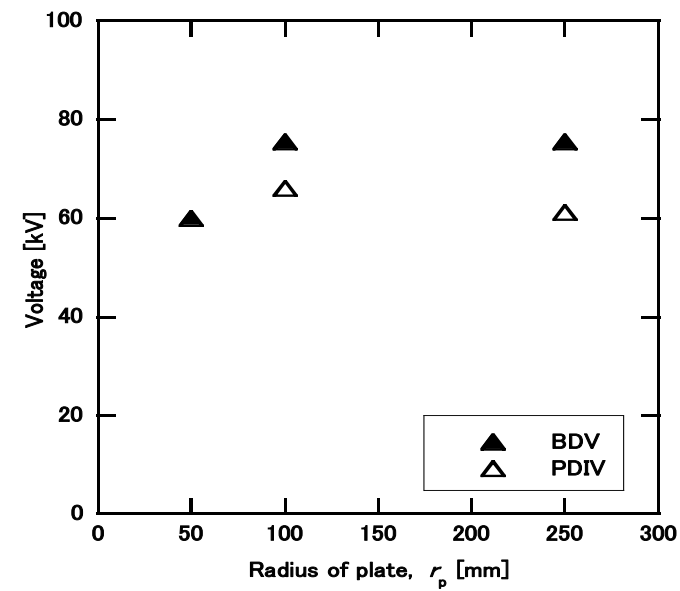

Figure 16. Relation between positive impulse breakdown voltage and the radius of the plate. $\boldsymbol{r}_{\mathrm{e}}=25(\mathrm{~mm})$ and $\boldsymbol{t}=11(\mathrm{~mm})$.

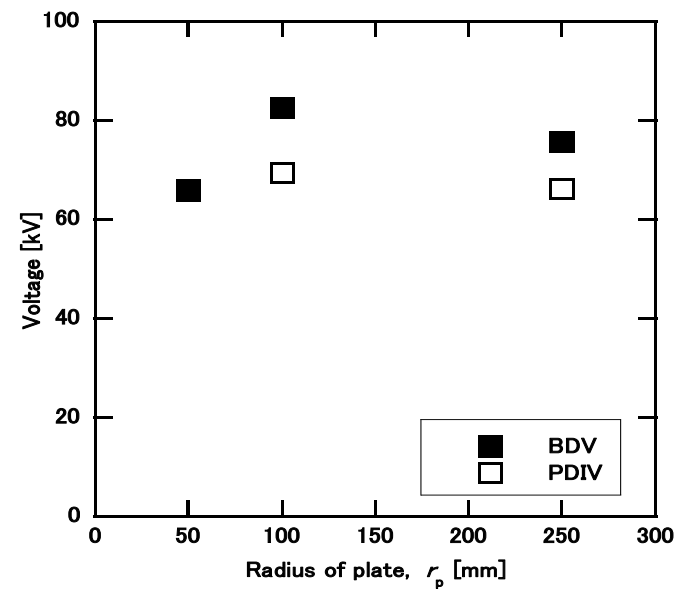

Figure 17. Relation between negative impulse breakdown voltage and the radius of the plate. $\boldsymbol{r}_{\mathrm{e}}=25(\mathrm{~mm})$ and $\boldsymbol{t}=11(\mathrm{~mm})$.

\section{DISCUSSION \\ 5.1 PARTIAL DISCHARG INCEPTION}

Figure 18 shows the relation of the partial discharge inception electric field (PDE) and the radius of embedded electrode, $\boldsymbol{r}$. PDIE wartalcurated from the measured PDIV in Figure 9 and the field ealculation results. As a result, PDIE is almost constant and is expected $3 \mathrm{kV} / \mathrm{mm}$. In other cases where $\boldsymbol{r}_{\mathrm{p}}$ of was varied, PDIE calculated from PDIV measured values on Figures 10 and 11 was also almost the same and was around $3 \mathrm{kV} / \mathrm{mm}$. The value of PDIE is reasangble because the discharge inception electric field in air is knowy around $30 \mathrm{kV} / \mathrm{cm}$.

From the above, it is deduced that the partial discharge will be initiated when the electric field reaches to be $3 \mathrm{kV} / \mathrm{mm}$ and the partial discharge inception voltage, PDIV can be increased with choosing the radius of embedded electrode appropriately. AC PDIV is quite important as the reason why the solid insulation material such as epoxy resin will be deteriorated by the partial discharge. In the actual switchgear, the partial discharge free design under the service voltage is necessary.

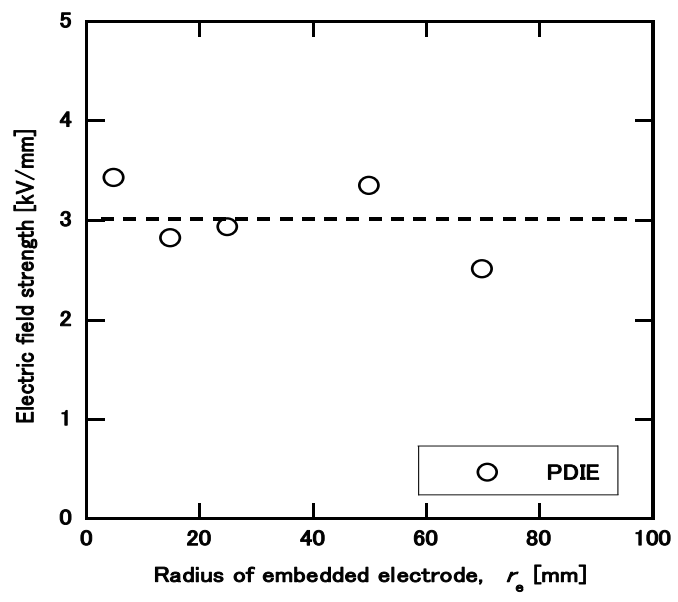

Figure 18. Relation between the partial discharge inception field strength and the radius of the embedded electrode under ac voltage application, $\boldsymbol{r}_{\mathbf{p}}=$ $100(\mathrm{~mm})$ and $\boldsymbol{t}=11(\mathrm{~mm})$

\subsection{DESJGN Of EMBEDDED ELECTRODE}

From Figures 9 and the characteristics of BDV and ac PDIV and are eployted in Figure 19. Because impulse BDV of positive polarity was smaller than that of negative polarity, impulse BSV of positive polarity was used. As mentioned in the sections 4.2 and 4.3, the radius of embedded electrode when BDV has the maximum value is different between ac and impulse. Therefore, the size of embedded electrode should be agedded in comparison with the voltage to be applied. If $\boldsymbol{r}_{\mathrm{e}}$ is lected appropriately in the range between 25 to $50 \mathrm{~mm}$ under the condition where $\boldsymbol{r}_{\mathbf{p}}=100(\mathrm{~mm})$, high insulation performance is expected.

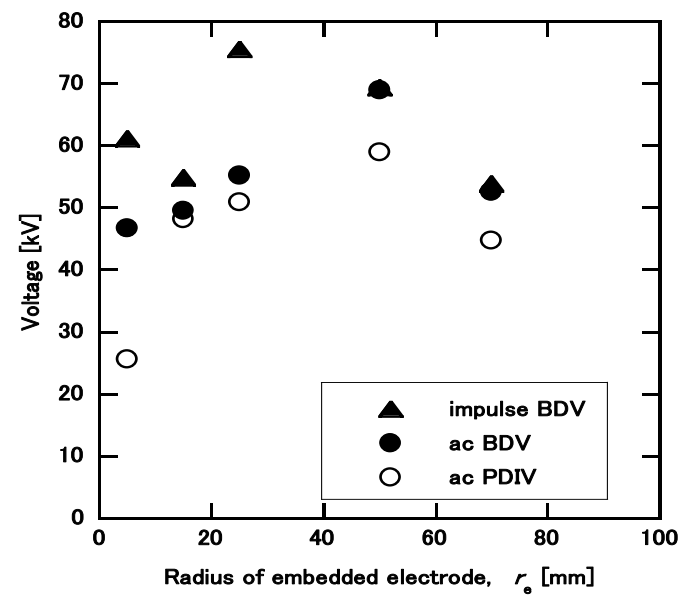

Figure 19. Relation between insulation performance and the radius of the embedded electrode.

Generally, the surface breakdown voltage (BDV) can be decided based on the surface distance, distributed electrostatic capacity per unit area and the voltage polarity. However, it is complicated in the present case. The surface discharge propagates from the partial discharge inception point in both directions (towards the center electrode and the ground electrode). The polarity of each surface discharge will be 
different and the electrostatic property of each surface is also different. Moreover, the potential at the starting point varies with the configuration of the test sample. Therefore, detailed discussion is necessary to decide the BDV. The estimation of BDV will be reported precisely in another paper.

\section{CONCLUSION}

Solid insulated switchgear (SIS) has been developed as substitution for medium voltage $\mathrm{SF}_{6}$ gas insulated switchgear. For further miniaturization and higher stress design, improvement of insulation performance is required. In SIS, solid/gas insulation system is composed. The breakdown strength along the surface is lower than that of solid. Then, the authors investigated the surface insulation performance using the test sample, which was the disc-shaped epoxy resin plate with embedded electrode. The sizes of embedded electrode and the plate were varied and the partial discharge inception voltage and the surface breakdown voltage were measured. The partial discharge inception position was also observed. The results are summarized as follows.

(1) Partial discharge is initiated at the point where the electric field strength along the surface has the maximum.

(2) The partial discharge inception electric field is almost the same regardless of the sizes of the plate and embedded electrode except for some conditions. The partial discharge inception electric field strength for ac voltage is almost the same as $3 \mathrm{kV} / \mathrm{mm}$.

(3) In case the radius of embedded electrode is varied, the surface breakdown voltage has a peak value. Howe ep, the radius where the breakdown voltage has the max mum is different between ac and impulse.

(4) The surface breakdown voltage increases with the thickness of the plate.

(5) The effect of the surface distance on the surface insulation performance is limited.

From the above, it is deduced that the surface insulation performance can be improved by choosing the size of embedded electrode appropriately. Furthermore, increasing the thickness of the solid materiatyis consulered to be effective.

\section{REFERENCES}

[1] J. Sato, S. Kino hifa, Sakaguchi, M. Miyagawa, T. Shimizu and M. Homma, "golid Insulated Switchgear and Investigation of its Mechanica and Electrical Reliability", Trans. IEE Japan, Vol.128-B, No.12, pr.1431-1438, 2008 (in Japanese).

[2] I. Yoshida T. Yano, N. Tohya, N. Inoue, M. Arioka, S. Sato, T. Toshie, The Development and Practical Use of a New 24 kV Dry Air Insulated Switchgear", Trans. IEE Japan, Vol.126, No.3, pp.359-364, 2006 (in Japanese).

[3] T. Rokunohe, Y. Yagihashi, F. Endo, K. Aoyagi, H. Saitoh and T. Oomori, "Development of $72 \mathrm{kV}$ High Pressure Air-insulated GIS with Vacuum Circuit Breaker", Trans. IEE Japan, Vol.125, No.12, pp.12701277, 2005 (in Japanese).

[4] S. Sato, K. Koyama, T. Itotani, S. Miyamoto and T. Maruyama, "Development of Vacuum Insulation Technology for a Multifunctional Vacuum Interrupter Loaded into an $\mathrm{SF}_{6}$ Gas Free $24 \mathrm{kV}$ Switchgear", Trans. IEE Japan, Vol.123, No.4, pp.442-449, 2003 (in Japanese).
[5] T. Shioiri, J. Sato, T. Ozaki, O. Sakaguchi, T. Kamikawaji, M. Miyagawa, M. Homma, K. Suzuki, "Insulation Technology for Medium Voltage Solid Insulated Switchgear", IEEE Conf. Electr. Insul. Dielectr. Phenomena, pp.341-344, 2003.

[6] J. Sato, O. Sakaguchi, N. Makishima, S. Kinoshita, T. Shioiri, T. Yoshida, M. Miyagawa, M. Homma and E. Kaneko, "New Technology for Medium Voltage Solid Insulated Switchgear", IEEE PES T\&D Conf., Vol.3, pp.1791-1796, 2002.

[7] e.g. T. Yoshida, H. Nishikawa, K. Takagi, S. Imakoma and N. Masaki, "Air Gap Flash-over Voltage Rise Due to Inserting a Barrier Plate", Trans. IEE Japan, Vol.B-100, No.1, pp.57-64, 1980 (in Japanese).

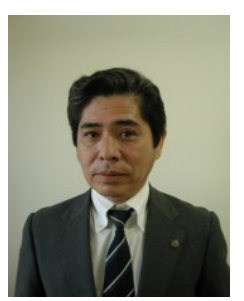

Takahiko Yamashita (M’00) was born in 198 in Fukuoka, Japan. He received B.E. M.E and D.E. degrees from Kyushu Universia, Japan, in 1980, 1982 and 1985 , respectively. He has been working in Nagasaki University, Japan, sinco 1985. He is a Professor of the Graduate Schoor f Engineering and a Vice President of Naga divi Undersity. He is a senior member of Institute of Electrieal Engineers Japan.

\section{1}

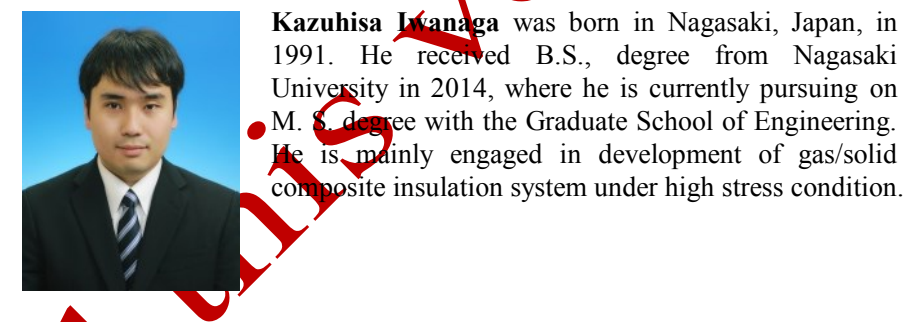

Kazuhisa IKanaga was born in Nagasaki, Japan, in 1991. He received B.S., degree from Nagasaki University in 2014, where he is currently pursuing on M. degree with the Graduate School of Engineering. He is mainly engaged in development of gas/solid Tomohiro Furusato (M'11) was born in Kagoshima, 3 Japan, in 1988. He received B.S., M.S., and Ph.D. degrees from Kumamoto University, Japan, in 2011, 2012, and 2014, respectively. He was with the JSPS, Kumamoto University, from 2013 to 2014, as a Research Fellow. Since 2014, he has been an Assistant Professor in Nagasaki University, Japan. His research interests are pulsed-power, creeping discharge, and discharge phenomena in supercritical fluids.

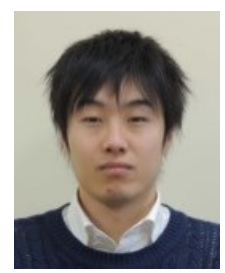

Hiroyuki Koreeda was born in Miyazaki, Japan, 1985. $\mathrm{He}$ received B.S. and M.S. Eng. degrees in electrical engineering from Nagasaki University, Nagasaki Japan, in 2008 and 2010. He was with the Mitsumi Electric Co. Ltd.. from 2010 to 2013. Since 2014, he has been working as a technician in Nagasaki University.

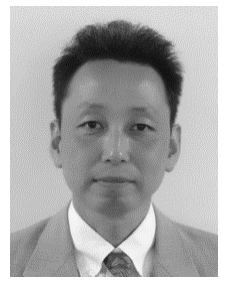

Tomoyuki Fujishima was born in 1966 in Fukuoka, Japan. He received B.E., M.E. and D.E. degrees from Kumamoto University, Japan, in 1989, 1991 and 1994, respectively. $\mathrm{He}$ has been working in Nagasaki University since 1994 and is an Associate Professor. His research interests are ozone generation, ozonic environment application and lightning protection. He is a member of the Institute of Electrical Engineers of Japan and the Japan Society of Applied Physics.

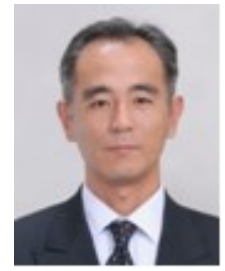

Junichi Sato was born in 1967 in Fukuoka, Japan. He received B.E., M.E. and D.E. degrees from Nagasaki University, Japan, in 1990, 1992 and 2011, respectively. $\mathrm{He}$ has been working in Toshiba Corporation, Tokyo, Japan since 1992. He belongs to the power and industrial systems $R \& D$ center, Toshiba Corporation. His research interests are solid and solid/gas composite insulation systems, vacuum interrupter and solid insulation switchgear. 